\title{
Cooperative Path Planning for a Class of Carrier-Vehicle Systems.
}

\author{
Emanuele Garone ${ }^{\circ}$, Roberto Naldi ${ }^{\dagger}$, Alessandro Casavola ${ }^{\circ}$ and Emilio Frazzoli ${ }^{\ddagger}$ \\ ○ Università della Calabria, Rende (CS), 87036, Italy. \\ $\dagger$ Università di Bologna, Bologna, 40133, Italy. \\ $\ddagger$ Massachusetts Inst. of Technology, Cambridge, MA, 02139, USA.
}

\begin{abstract}
In this work we concentrate on the problem of path planning in a scenario in which two different vehicles with complementary capabilities are employed cooperatively to perform a desired task in an optimal way. In particular we consider the case in which a vehicle carrier, typically slow but with virtually infinite operativity range, and a carried vehicle, which on the contrary is typically fast but with a shorter operative range, can be controlled together to pursuit a certain mission while minimizing a pre-defined cost function. In particular we will concentrate on a particular scenario, which we denoted as "fast-rescue" problem, providing optimal and heuristic solutions to various cases.
\end{abstract}

\section{INTRODUCTION}

The complexity of many applications envisioned for future autonomous vehicle networks, ranging from planetary exploration to security missions, requires a broad range of capabilities for individual units-ranging from air, ground or sea mobility, to sophisticated multi-modal sensor suites and actuation devices - which cannot be implemented on a single platform class. Rather, it may be necessary to coordinate diverse specialized units to attain complex objectives in a reliable, timely, and efficient fashion [14]. While considerable progress has been made on cooperative control of networks of homogeneous vehicles (see for example [1], [2], [6], [8], heterogeneous networks are still relatively poorly understood. In particular, it is of interest to understand how to optimally exploit the different capabilities of individual vehicles.

In this paper, we concentrate on a very simple system of heterogeneous vehicles, arising from the combination of (i) a slow autonomous surface carrier (typically a ship), with long range operational capabilities, and (ii) a faster vehicle (typically an helicopter, an UAV or an offshore vehicle) with a limited operative range. The carrier is able to transport the faster vehicle, as well as to deploy, recover, and service it. Even though this two-vehicle system is very simple, many interesting problems can be stated, involving optimization and coordination problems [7], [12], [13].

Here we will deal mostly with the so-called "fast rescue problems." By "fast rescue problems" we mean those scenarios in which one (or eventually many) "targets" with known and non-changing position have to be visited in the shortest possible time. This is the case of rescue or event monitoring

Corresponding author: Roberto Naldi, CASY-DEIS University of Bologna, Via Risorgimento 2, 40136 Bologna, Italy. Tel: 00390512093875 , Fax:0039051 2093073, email: roberto.naldi@unibo.it missions in which it is important to arrive quickly at one or more desired locations.

As a preliminary work, in this paper we studied such problems by assuming holonomic dynamic models to represent the behavior of both the carrier and carried vehicles.

The paper is organized as follows. Section 2 introduces the carrier-vehicle systems, its dynamics and the constraints related. In Paragraph 3 the rescue problems are stated and in Paragraph 4 some solutions to the most common cases are proposed. Finally some conclusion and some indication on future research end the paper.

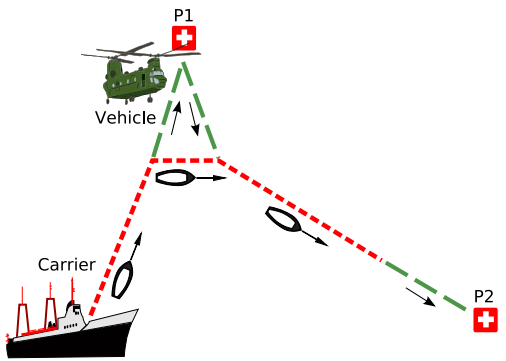

Fig. 1. The carrier-vehicle system on a rescue scenario.

\section{The CARrier-Vehicle Hybrid System}

The system we are going to deal with is composed by two different vehicles, a vehicle carrier (also denoted in the following as carrier), whose variables and functions will be denoted by subscript ${ }_{c}$, and a carried vehicle, denoted by subscript ${ }_{v}$. In the following we will refer to the combined system as the carrier-vehicle system.

To derive a mathematical model for the system, we will consider the vehicles as points belonging to the Euclidean space $\mathbb{R}^{2}$. The admissible path followed by each vehicle is a continuous curve $\Gamma:\left[0, t_{f}\right] \mapsto \mathbb{R}^{2}$. Let us fix an inertial frame $F_{i}=\left\{O_{i}, \vec{i}, \vec{j}\right\}$ and define with the notation $v^{i}$ vectors $v \in \mathbb{R}^{2}$ expressed in $F_{i}$. The following state variables are introduced

$$
p_{c}=\left[\begin{array}{ll}
x_{c}^{i} & y_{c}^{i}
\end{array}\right]^{T} \quad p_{v}=\left[\begin{array}{ll}
x_{v}^{i} & y_{v}^{i}
\end{array}\right]^{T} .
$$

with $p_{c}(t) \in \mathbb{R}^{2}, p_{v}(t) \in \mathbb{R}^{2}$ respectively the position of the vehicle carrier and the vehicle at time $t$ in the inertial frame $F_{i}$. We will assume that the position of the carrier $p_{c}(t)$ evolves accordingly to the first order O.D.E

$$
\dot{x}_{c}=V_{c} \cos \left(\phi_{c}\right) \quad \dot{y}_{c}=V_{c} \sin \left(\phi_{c}\right)
$$


with $V_{c} \in \mathbb{R}^{+}$the fixed velocity of the carrier and $\phi_{c} \in \mathbb{R}$ the control input.

To model the dynamics of the carried vehicle we distinguish between two different situations:

1) when it is not carried it evolves following its free behavior:

$$
\dot{x}_{v}=V_{v} \cos \left(\phi_{v}\right) \quad \dot{y}_{v}=V_{v} \sin \left(\phi_{v}\right)
$$

with $V_{v} \in \mathbb{R}^{+}, V_{v}>V_{c}$ and $\phi_{v} \in \mathbb{R}$ the control input for the vehicle.

2) when it is carried is a function of the carrier dynamics and in particular it will coincide with the carrier position, $p_{v}(t)=p_{c}(t)$.

From the above arguments it appears that the carried vehicle dynamics show an intrinsically hybrid behavior. For such a reason let us introduce a binary variable $c(t) \in\{0,1\}$ with the following semantic:

1) $c(t)=1$ if the vehicle is carried;

2) $c(t)=0$ if it is not carried.

By means of $c(t)$ we define a 2 states automaton (see also [17] $)(c(t)=0$ and $c(t)=1)$ that changes his status accordingly to the following guard conditions:

1) an input command, $u_{w}(t) \in\{0,1\}$, that denote the "will" of change the actual state $c(t)$;

2) "compatibility constraints" that denote the conditions in term of system's state under which approach of the two vehicles are possible, nominally $p_{c}(t)=p_{v}(t)$.

Since one of the distinguish feature of the carried-vehicle is to have a finite operativeness (e.g. fuel), we introduce the internal dynamics

$$
\dot{a}(t)= \begin{cases}-1 & \text { if } c(t)=0 \\ 0 & \text { if } c(t)=1\end{cases}
$$

where $a \in \mathbb{R}$ is the operativeness of the vehicle.

The operativeness of the vehicle is indeed decreasing when the vehicle is not carried $(c(t)=0)$, since it is assumed it has to use its own power, while it remains constant when the vehicle is on the deck of the carrier $(c(t)=1)$. For the sake of simplicity, it is supposed that anytime an approach occurs, $a(t)$ is restored to a certain constant default value $\bar{a}$. The faster vehicle is constrained to have non-negative operativeness; if this condition fails to be true an event occurs which bring the system to a fault state. This faulty situation is captured by adding a further state to the automaton describing the vehicle's dynamics, i.e. $c(t)=-1$ (faulty state).

\section{The Rescue Problems FAmily}

In this paper we are interested in studying and provide solutions for the so called "fast rescue problems. By fast rescue problems we mean those missions in which an ordered collection of $n$ points, namely $q_{1}, \ldots, q_{n}$ where $q_{i} \in \mathbb{R}^{2}$, have to be visited ordinately in the "shortest possible time" by the fast vehicle, eventually satisfying a prescribed takeoffvisit-landing sequence.
More formally, if we define by $t_{i} \in \mathbb{R}^{+}$the time in which $q_{i}$ is visited, we can state the general rescue problem in the following form:

Given a carrier-vehicle system with initial conditions $p_{c}(0)=p_{v}(0)=p_{c, 0} \in \mathbb{R}^{2}$ and $c(0)=1$, and a set of objective points $\hat{q}_{\text {list }}=\left\{q_{1}, q_{2}, \ldots ., q_{n}\right\}$ with $q_{1} \in \mathbb{R}^{2}, i=$ $1, \ldots, n$

find the optimal inputs $(\hat{u}(t))_{t:[0, \infty)}$

minimizing a certain objective function obj $\left(t_{1}, \ldots, t_{n}\right)$

such that a certain given sequence of $c(t)$ state changes and visits events is satisfied

and eventually the vector $\hat{t}=\left[\begin{array}{lll}t_{1} & \ldots & t_{n}\end{array}\right]$ belongs to a certain admissible set $T$

where $\hat{u}(t)=\left[\phi_{c}(t), \phi_{v}(t), u_{w}(t)\right]^{T}$ is the vector representing all the inputs of the system.

In the next section we will introduce and solve some problems belonging to this family.

\section{Some SOlution}

\section{A. Fastest approach to a point}

Given an initial point $p_{0}$ and a point to reach $q_{1}$, we want to find a trajectory for the carrier-vehicle system described above such that $t_{1}$ is minimized. Because the time spent in any trajectory between two points by each one of the two vehicles can be always equal or grater of the time to follow a straight line (we recall that the admissible path is a continuous curve), the problem can be reshaped into finding a take-off point $p_{t o}$ such that the carried vehicle has enough operativeness to take-off at $p_{t o}$, and to reach $q_{1}:\left\|p_{t o}-q_{1}\right\|_{2} \leq V_{v} a$.

A formal definition of the problem is then

$$
\left\{\begin{array}{l}
\min _{p_{0}} t_{1} \\
\frac{\left\|p_{t o}-q_{1}\right\|_{2}}{V_{v}} \leq \bar{a} \\
\frac{\left\|p_{o}-p_{t o}\right\|_{2}}{V_{c}}+\frac{\left\|p_{t o}-q_{1}\right\|_{2}}{V_{v}} \leq t_{1}
\end{array}\right.
$$

A straightforward lower bound on the cost, that is valid in all the missions we will introduce here, is the optimal path of a vehicle that can always go at velocity $V_{c}$ and it is allowed for certain amount of time $(\bar{a})$ to go at velocity $V_{v}$. Such a fastest path is obtained by means of a straight line between the starting point $p_{0}$ and the arrival point $q_{1}$ such that for the maximum allowed time $\bar{a}$ it goes to velocity $V_{v}$, and for the remaining time at velocity $V_{c}$, i.e.

$$
=\min \left\{\bar{a}, \frac{\left\|p_{0}-q_{1}\right\|_{2}}{V_{v}}\right\}+\max \left\{0, \frac{\left\|p_{0}-q_{1}\right\|_{2}-V_{v} \bar{a}}{V_{c}}\right\}
$$

The optimal solution for problem (5) is then

$$
p_{t o}=\left\{\begin{array}{l}
p_{0} \quad \text { if } \frac{\left\|p_{0}-q_{1}\right\|_{2}}{V_{v}} \leq \bar{a} \\
p_{0}+V_{v} \bar{a}\left(q_{1}-p_{0}\right)
\end{array}\right.
$$

since it's cost coincides with the lower bound (6). 
The simple geometric intuition behind such a solution is the following.

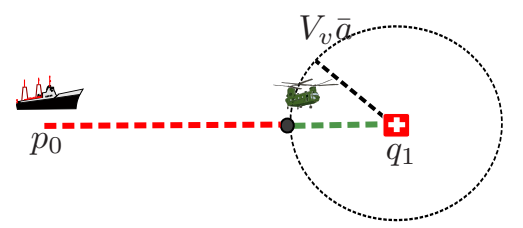

Fig. 2. Geometry of the fastest approach to a point problem.

The carried object can be launched only after that the vehicle is inside a circle of radius $V_{v} \bar{a}$. Then, because carried vehicle is faster of the carrier, as soon as the carrier is inside this circle, it has to launch the carried one that has to follow a straight line to the objective. It is trivial to see that no other choice is faster then this one. Moreover by simple geometric consideration, the shortest path for the carrier to approach the circle is a straight line directed to its center.

Remark - Notice that since $V_{v}>V_{c}$, the optimal solution always impose $c(t)=-1, \forall t>t_{1}$, The reason is that it has to use all the operativeness $a$ to reach $q_{i}$ as fast as possible. This implies that there is no fuel to come back to the carrier that being slower can't arrive in that point at the same time.

\section{B. Fastest approach to a point and re-entry mission}

Given an initial point $p_{0}$ and a point to reach $q_{1}$ we want to find a trajectory for the carrier-vehicle system described above such that the arrival time at $q_{1}$ is minimized and, after the point is visited, the fast vehicle is able to come back to the carrier. The problem can be reshaped in finding a takeoff point $p_{t o}$ and the landing point $p_{l}$ and trajectories such that the following constraints are satisfied:

1) the carried vehicle trajectory is such that it has enough operativeness to take-off at $p_{t o}$, to reach the objective and to arrive in $p_{l}$. This implies that the points have to be such that $\left\|p_{t o}-q_{1}\right\|_{2}+\left\|q_{1}-p_{l}\right\|_{2} \leq V_{v} \bar{a}$;

2) the carrier vehicle trajectory is such that it is able to arrive from $p_{t o}$ to $p_{l}$ in a time that is not greater than the maximum operativeness $\bar{a}$. This implies that the two points have to be at distance $\left\|p_{t o}-p_{l}\right\|_{2} \leq V_{c} \bar{a}$.

We want to minimize the "safe" arrival time $t_{l}$ which is the sum of two contributions:

1) the time $t_{1,0}$ used by the carrier vehicle to reach $p_{t o}$ from $p_{0}$. Because for such a kind of vehicle the fastest path is always the straight line, $t_{1,0}=\frac{1}{V_{c}}\left\|p_{0}-p_{t 0}\right\|_{2}$;

2) the time $t_{2,0}$ used by the carried vehicle to arrive at $q_{1}$. Again the fastest path is the straight line then $t_{1,1}=$ $\frac{1}{V_{v}}\left\|p_{t 0}-q_{1}\right\|_{2}$.

As a consequence we can reduce the core of the problem to the following optimization problem

$$
\left\{\begin{array}{l}
\min \frac{1}{V_{v}}\left\|p_{t 0}-q_{1}\right\|_{2}+\frac{1}{V_{c}}\left\|p_{0}-p_{t 0}\right\|_{2} \\
\left\|p_{t o}-q_{1}\right\|_{2}+\left\|q_{1}-p_{l}\right\|_{2} \leq V_{v} \bar{a} \\
\left\|p_{t o}-p_{l}\right\|_{2} \leq V_{c} \bar{a}
\end{array}\right.
$$

Even in this case is possible to avoid numerical solutions and instead to find a closed form solution. Let us start from the geometry of the problem.

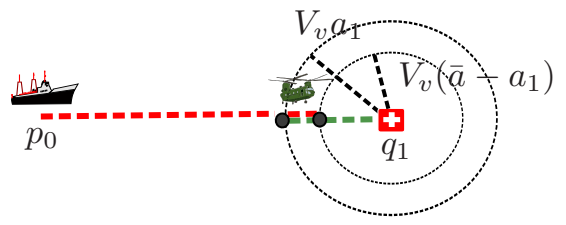

Fig. 3. Geometry of the fastest approach to a point an re-entry mission problem.

By exploiting the symmetry of the "operativeness circle", we know that the carried vehicle will be launched when it will enter in a certain circle of radius $V_{v} a_{1} \leq V_{v} \bar{a}$. From that point the launched vehicle will follow the fastest trajectory to $q_{1}$, i.e. the straight line. Exactly like in the previous case, the optimal path for the carrier between the starting and the launching points is the straight line between $p_{0}$ and $q_{1}$. It remains now to discuss the choice of $a_{1}$ and how to build a feasible re-entry trajectory.

The main idea is that the optimum is obtained if the time to follow the straight line between $p_{o}$ and $q_{1}$ is minimized, or, equivalently, if we choose the maximum $a_{1}$ such that the recovery is possible. Then, the carrier at time $t_{1,0}+\bar{a}$ has to be as near as possible to $q_{1}$. For such a reason it will continue on the straight line to $q_{1}$. By following this idea, we have then

$$
a_{1}=\min \left\{\frac{\left(V_{c}+V_{v}\right)}{2 V_{v}} \bar{a}, \frac{\left\|p_{0}-q_{1}\right\|_{2}}{V_{v}}\right\}
$$

Remark - This is because the space the carrier will cover during the flight of the carried vehicle $V_{c} \bar{a}$ has to be equal to the difference of space between the "go" and the "come back" path of the launched vehicle i.e. $V_{v} a_{1}-V_{v}\left(\bar{a}-a_{1}\right)=$ $2 V_{v} a_{1}-V_{v} \bar{a}$.

The only further case to be analyzed is when $q_{1}$ is already inside the circle of radius $a_{1}$, in which trivially there is an instantaneous take-off. From the above discussion it follows that the minimum time to reach the objective $q_{1}$ is

$$
\begin{aligned}
t_{1}= & \max \left\{0, \frac{\left\|p_{0}-q_{1}\right\|_{2}}{V_{c}}-\frac{V_{c}+V_{v}}{2 V_{c}} \bar{a}\right\}+ \\
& +\min \left\{\frac{V_{c}+V_{v}}{2 V_{v}} \bar{a}, \frac{\left\|p_{0}-q_{1}\right\|_{2}}{V_{v}}\right\} .
\end{aligned}
$$

\section{Fastest approach to 2 far points with re-entry mission}

Let us consider the case in which the carried vehicle has to reach two different points in an ordered way, minimizing the time $t_{2}$ and providing that, after each visit, it comes back to the carrier. Hereafter, in order to avoid the analysis of cases of low practical interest, it will be supposed that the starting point and the two points to reach are "far enough" with respect to the carried vehicle operativeness, nominally we have $d_{0,1}=\left\|q_{1}-p_{0}\right\|_{2}>V_{v} \bar{a}$. and $d_{1,2}=\left\|q_{2}-q_{1}\right\|_{2}>$ $2 V_{v} \bar{a}$.

Because the shortest path between two different points in the Euclidean space $\mathbb{R}^{2}$ is represented by the straight line and 
because both the vehicles have the ability to "wait in a point", it is possible to recast the original problem of finding the optimal trajectory as the problem of fixing 4 (distinguished) points: the two take-off points $p_{t o 1}, p_{t o 2}$ and the two landing points $p_{l 1}, p_{l 2}$. We can then state the following optimization problem:

$$
\begin{aligned}
& \min t_{p_{0}, p_{t o 1}}+t_{p_{t o 1}, p_{l 1}}+t_{p_{l 1}, p_{t o 2}}+t_{p_{t o 2}, q_{2}} \\
& \frac{\left\|p_{0}-p_{t o 1}\right\|_{2}}{V_{c}}=t_{p_{0}, p_{t o 1}} \frac{\left\|p_{t o 1}-p_{l 1}\right\|_{2}}{V_{c}} \leq t_{p_{t o 1}, p_{l, 1}} \\
& \frac{\left\|p_{t o, 1}-q_{1}\right\|_{2}}{V_{v}}+\frac{\left\|p_{t o 1}-q_{1}\right\|_{2}}{V_{v}} \leq t_{p_{t o 1}, p_{l 1}} \\
& \frac{\left\|p_{l 1}-p_{t o 2}\right\|_{2}}{V_{c}}=t_{p_{l 1}, p_{t-o, 2}} \frac{\left\|p_{t o 2}-q_{2}\right\|_{2}}{V_{v}} \leq t_{p_{t o 2}, q_{2}} \\
& \frac{\left\|q_{2}-p_{l 2}\right\|_{2}}{V_{v}} \leq t_{q_{2}, p_{l 2}} \quad \frac{\left\|p_{l, 2}-p_{t o, 2}\right\|_{2}}{V_{c}} \leq t_{p_{t o, 2}, p_{l, 2}} \\
& 0 \leq t_{p_{t o 1}, p_{l 1}} \leq \bar{a} \quad 0 \leq t_{p_{t o 2}, p_{l 2}} \leq \bar{a} \\
& 0 \leq t_{p_{t o 2}, q_{2}}+t_{q_{2}, p_{l 2}} \leq \bar{a}
\end{aligned}
$$

With an eye at (10), we observe that the last two terms of the cost function represent the objective of the 1-point fastest approach problem (8) with an initial condition that corresponds to the given landing point $p_{l 1}$. For this reason the last term of the objective function can be optimized independently, allowing to further reduce the problem to the determination of two points $p_{t o 1}$ and $p_{l 1}$, i.e.

$$
\begin{aligned}
& \min t_{p_{0}, p_{t o 1}}+t_{p_{t o 1}, p_{l 1}}+t_{q 2} \\
& \quad \frac{\left\|p_{0}-p_{t o 1}\right\|_{2}}{V_{c}}=t_{p_{0}, p_{t o 1}} \frac{\left\|p_{t o 1}-p_{l 1}\right\|_{2}}{V_{c}} \leq t_{p_{t o 1}, p_{l, 1}} \\
& \frac{\left\|p_{t o 1}-q_{1}\right\|_{2}}{V_{v}}+\frac{\left\|p_{t o 1}-q_{1}\right\|_{2}}{V_{v}} \leq t_{p_{t o 1}, p_{l 1}} \\
& \frac{\left\|p_{l 1}-q_{2}\right\|_{2}}{V_{c}}-\frac{V_{c}+V_{v}}{2 V_{c}} \bar{a}+\frac{V_{c}+V_{v}}{2 V_{v}} \bar{a}=t_{q 2} \\
& 0 \leq t_{p_{t o 1}, p_{l 1}} \leq \bar{a}
\end{aligned}
$$

The solution of such an optimization problem can be computationally hard. For such a reason, geometric solutions (eventually sub-optimal) have been investigated. In order to proceed we first derive an upper bound and two distinguished lower bounds to be used in the analysis.

An upper bound to the optimal cost can be found considering the case in which the carried vehicle never takes off (or equivalently has an instantaneous take-off and landing) and goes straight to the first point. Then, to reach the last point, it follows the optimal 1-point strategy with cost given by (9). The overall cost of this solution is given by

$$
t_{u p}=\frac{d_{0,1}}{V_{c}}+\frac{d_{1,2}}{V_{c}}-\bar{a}\left(\frac{V_{c}+V_{v}}{2 V_{c}}\right)+\bar{a}\left(\frac{V_{c}+V_{v}}{2 V_{v}}\right) .
$$

Remark - The upper bound (12) coincides with the optimal solution when the starting point $p_{0}$ and the objective points $q_{1}$ and $q_{2}$ are placed on the same line.

A first lower bound to the optimal cost can be calculated assuming that the trajectory of the system is characterized by a speed $V_{v}$ for $\bar{a}$ seconds, in the shortest-path between $p_{0}$ and $q_{1}$, and a speed $V_{c}$ for the remaining time. Again from $q_{1}$ to the second objective $q_{2}$ we can make use of the 1-point optimal solution (9). The cost of such a lower bound is

$t_{\text {low }}=\frac{d_{0,1}}{V_{c}}+\bar{a}\left(1-\frac{V_{v}}{V_{c}}\right)+\frac{d_{1,2}}{V_{c}}-\bar{a}\left(\frac{V_{c}+V_{v}}{2 V_{c}}\right)+\bar{a}\left(\frac{V_{c}+V_{v}}{2 V_{v}}\right)$.

A second straightforward lower bound is the cost of the 1point optimal solution between $p_{0}$ and $q_{2}$, discarding the fact we need to visit $q_{1}$. Such a lower bound has cost:

$$
t_{\text {low }}=\frac{\left\|q_{2}-p_{0}\right\|_{2}}{V_{c}}-\bar{a}\left(\frac{V_{c}+V_{v}}{2 V_{c}}\right)+\bar{a}\left(\frac{V_{c}+V_{v}}{2 V_{v}}\right)
$$

In many significant cases it is possible to find a geometric solution whose cost is equal to those two lower bounds, and then optimal.

The main idea, underling the geometric constructions that we will present here, is to take advantage of the possibility of the carrier to launch the carried vehicle (that will visit $q_{1}$ ) and to take a shortcut in order to have a rendezvous somewhere on the straight line to $q_{2}$ before the carried vehicle has exhausted the fuel. This idea is depicted in Fig. 4.

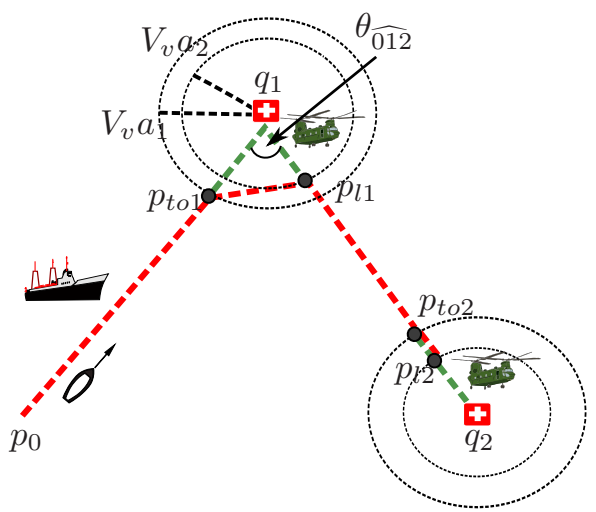

Fig. 4. The geometric construction behind Proposition 1.

The following propositions can be stated:

Proposition 1: Let $\theta_{\widehat{012}}$ be the smallest angle formed by the segments $\overline{p_{0} q_{1}}$ and $\overline{q_{1} q_{2}}$. If $\theta_{\widehat{012}} \leq \operatorname{acos}\left(1-2\left(V_{c}^{2} / V_{v}^{2}\right)\right)$ then it exists at least one optimal solution to the problem such that the total cost is equal to lower bound (13)

Proof. Let us proceed with a constructive proof. As shown in Fig. 4, the idea is that the carried vehicle will be released at distance $V_{v} a_{1}$ from $q_{1}$ on the line segment $\overline{p_{0} q_{1}}$ and will be recovered at distance $V_{v} a_{2}$ from $q_{1}$ on the straight path $\overline{q_{1} q_{2}}$. In order to satisfy the hypothesis of the lower bound (13) and the operativeness constraints,

$$
a_{1}+a_{2}=\bar{a}
$$

has to be imposed.

As it is clear from the Fig. 4, between the take-off and the landing events, the carrier will "cut the edge" by following the straight segment $\overline{q_{t o 1}, q_{l 1}}$ whose length will be denoted by $V_{c} t_{\text {carrier. }}$. Because of operativeness constraints, this proposed solution will be a feasible one to the given problem if

$$
t_{\text {carrier }} \leq \bar{a}
$$


By simple triangle consideration it is possible to see that $V_{c} t_{\text {carrier }} \leq V_{v} a_{1}+V_{v} a_{2}=V_{v} \bar{a}$. In particular by using Carnot theorem it is possible to write that

$$
\left(V_{c} t_{\text {carrier }}\right)^{2}=\left(V_{v} a_{1}\right)^{2}+\left(V_{v} a_{2}\right)^{2}-2 V_{v}^{2} a_{1} a_{2} \cos \left(\theta_{\overparen{012}}\right) \text {. }
$$

Because of the condition (15), it is of interest, for a given angle $\theta_{\widehat{012}}$, to choose $a_{1}$ and $a_{2}$ in order to minimize $t_{\text {carrier }}$. Let us rewrite (17) by using (15)

$$
\begin{aligned}
& \left(t_{\text {carrier }}\right)^{2}=\frac{1}{V_{c}}\left[\left(V_{v} a_{1}\right)^{2}+\left(V_{v}\left(\bar{a}-a_{1}\right)\right)^{2}+\right. \\
& \left.-2 V_{v}^{2} a_{1}\left(\bar{a}-a_{1}\right) \cos \left(\theta_{\widehat{012}}\right)\right]= \\
& =\frac{1}{V_{c}}\left[2 V_{v}^{2} a_{1}^{2}+V_{v}^{2} \bar{a}-2 V_{v}^{2} \bar{a} a_{1}-2 V_{v}^{2} \bar{a} a_{1} \cos \left(\theta_{\widehat{012}}\right)+\right. \\
& \left.-2 V_{v}^{2} a_{1}^{2} \cos \left(\theta_{\widehat{012}}\right)\right]
\end{aligned}
$$

Being $t_{\text {carrier }} \geq 0$, the minimum is reached when the minimum of $t_{\text {carrier }}^{2}$ is reached. Then by nullifying the derivative of (18) w.r.t. $a_{1}$, we have

$$
\left[4 a_{1}-2 \bar{a}-2 \bar{a} \cos \left(\theta_{\widehat{012}}\right)-4 a_{1} \cos \left(\theta_{\overparen{012}}\right)\right]=0 .
$$

Consequently the choice of $a_{1}$ and $a_{2}$ that minimize $t_{\text {carrier }}$ is

$$
a_{1}=a_{2}=\left(\frac{1+\cos (\theta)}{2+2 \cos (\theta)}\right) \bar{a}=\frac{1}{2} \bar{a}
$$

and the minimum $t_{\text {carrier }}$ is:

$$
t_{\text {carrier }}=\bar{a} \frac{V_{v}}{V_{c}} \sqrt{\frac{1}{2}-\frac{\cos \left(\theta_{\overparen{012}}\right)}{2}}
$$

By imposing condition (16) it is possible to find that the maximum angle for which this solution is feasible: $\sqrt{0.5\left(1-\cos \left(\theta_{\overparen{012}}\right)\right)}<V_{c} / V_{v}$. This inequality holds true if $\cos \left(\theta_{\widehat{012}}\right) \leq 1-2\left(V_{c}^{2} / V_{v}^{2}\right)$ that for $\theta_{\widehat{012}} \in[0, \pi)$ is $\theta_{\widehat{012}} \leq \operatorname{acos}\left(1-2 V_{c}^{2} / V_{v}^{2}\right)$

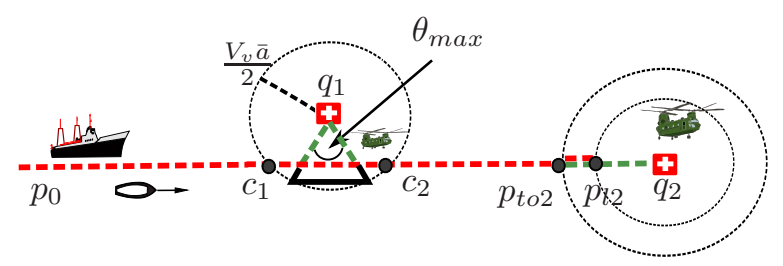

Fig. 5. The geometric interpretation behind Proposition 2.

Proposition 2: If the straight line between $p_{0}$ and $q_{2}$ touches in 2 points, respectively $c_{1}$ and $c_{2}$, the circle of center $q_{1}$ and radius $V_{v} \bar{a} / 2$ and if the smallest angle defined by the segments $\overline{c_{1} q_{1}}$ and $\overline{q_{1} c_{2}}$ is bigger or equal than $\operatorname{acos}\left(1-2 \frac{V_{c}^{2}}{V_{v}^{2}}\right)$ then it exists at least one solution such that the total cost is equal to lower bound (14) .

Proof. The carrier will go directly to the last point through a straight line. By hypothesis, such a straight line will intersect a triangle, built like the one in Fig. 5, with angle $\theta_{\max }=\operatorname{acos}\left(1-2 \frac{V_{c}^{2}}{V_{v}^{2}}\right)$. Following the proof of Proposition 1 , it is known that the time used by the carrier to follow the basis of that triangle is equal to the time the fast vehicle uses to follow the other two sides. Then using those intersection point as take-off and rendezvous point, a feasible solution of cost (14) is reached.

For the case not covered by the two proposition above some heuristics have been developed.

A first heuristic can be simply obtained by using the 1point solution. The idea is two perform two time a "single point" iteration. Such an idea it is very simple and has guaranteed results both for the 2-point cases and for the generalized one and will be described in the next subsection.

A second heuristic here proposed is built in the following way. Let us build a triangle with one vertex on $q_{1}$ and the other two on the circle of center $q_{1}$ and radius $\frac{1}{2} \bar{a} V_{v}$ and whose angle on $q_{1}$ is equal to $\theta_{\max }$, such that it is included in the angle $\theta_{\widehat{012}}$ and such that it does not intersect the segment $\overline{p_{0} q_{2}}$ as depicted in Fig. 6. If we fix the two points determined by the intersection of such a triangle and the circle as the take-off point and the rendezvous one

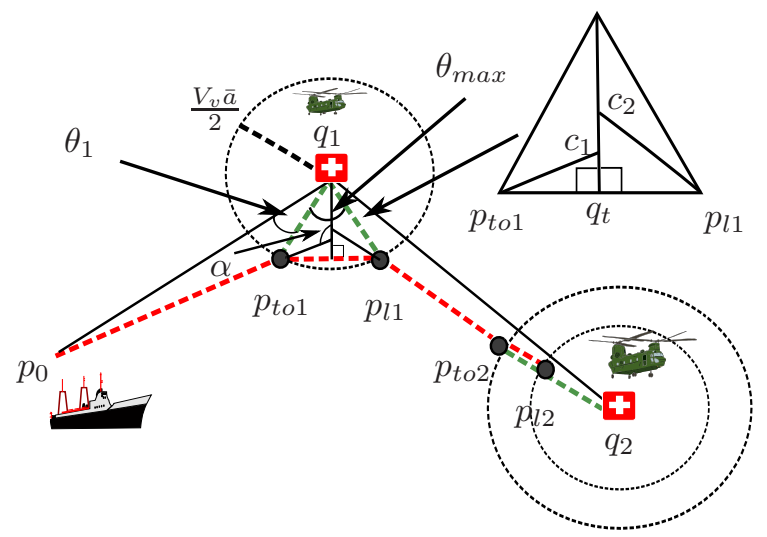

Fig. 6. The geometric interpretation behind the algorithm used for the 2 point case.

the total cost of the strategy will be

$$
\begin{aligned}
& t_{e u}=\frac{\sqrt{\left(\frac{1}{2} \bar{a} V_{v}\right)^{2}+d_{1}^{2}-\left(\bar{a} V_{v}\right) d_{1} \cos \left(\theta_{1}\right)}}{V_{c}}+\bar{a}+ \\
& +\frac{\left(\frac{1}{2} \bar{a} V_{v}\right)^{2}+d_{2}^{2}-\left(\bar{a} V_{v}\right) d_{2} \cos \left(\theta_{\widehat{012}}-\theta_{\max }-\theta_{1}\right)}{V_{c}}+ \\
& -\bar{a}\left(\frac{V_{c}+V_{v}}{2 V_{c}}\right)+\bar{a}\left(\frac{V_{c}+V_{v}}{2 V_{v}}\right)
\end{aligned}
$$

where $\theta_{1} \in\left[0, \theta_{\widehat{012}}-\theta_{\max }\right]$ is the one defined in Fig. 6 . In order to determine an optimal $\theta_{1}$, a simple numerical optimization can be performed, as in Fig. 7.

It is possible to prove that it always exist at least a choice of $\theta_{1} \in\left[\theta_{\widehat{012}}-\theta_{\max }\right]$ such that this solution, in the case Proposition $1 \& 2$ doesn't apply, it is always lower then the upper bound (12). For this purpose let us consider the particular solution $\theta_{1}=\left[\theta_{\widehat{012}}-\theta_{\max }\right] / 2$ as depicted in Figure 6, where, by construction, the angle denoted as $\alpha$ is always grater than $\pi / 2$

Then let us draw the lines $\overline{p_{0} p_{t o 1}}$ and $\overline{q_{2} p_{l 1}}$ until they touch the height segment of the triangle. Let us denote by $c_{1}$ and $c_{2}$ such an intersection point and by $q_{t}$ the center of the basis of the triangle. 


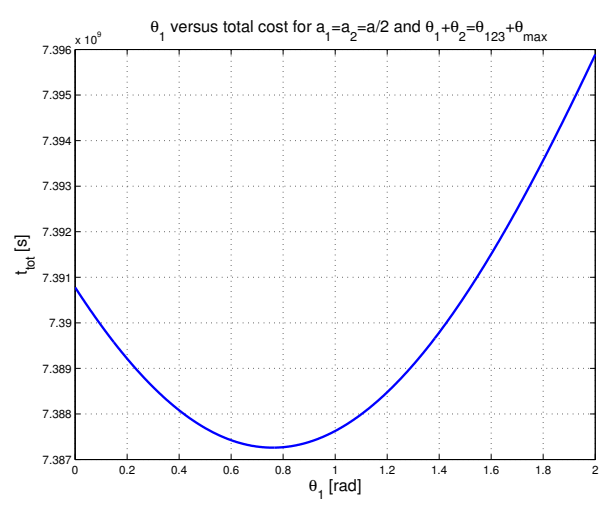

Fig. 7. The cost resulting from local search techniques applied to the free paramenter $\theta_{1}$.

Because $\alpha>\pi / 2$ then, from the properties of the triangles, $\left\|c_{1}-p_{0}\right\|_{2}<\left\|q_{1}-p_{0}\right\|_{2}$ and $\left\|c_{c}-p_{1}\right\|_{2}<$ $\left\|q_{2}-q_{1}\right\|_{2}$. Let us concentrate on $\left\|c_{1}-p_{0}\right\|_{2}<\left\|q_{1}-p_{0}\right\|_{2}$ (the same will be on the other inequalities, by symmetry). We observe that $\left\|p_{t o 1}-p_{0}\right\|_{2}+\left\|c_{1}-p_{t o 1}\right\|_{2}<\left\|q_{1}-p_{0}\right\|_{2}$.

Since $\left\|c_{1}-p_{t o 1}\right\|_{2}$ is the hypotenuse of a square triangle of basis $1 / 2 \bar{a} V_{c}$, we have also that $\frac{1}{2} \bar{a} V_{c}<\left\|c_{1}-p_{t o 1}\right\|_{2}$ and finally $\frac{1}{2} \bar{a} V_{c}+\left\|c_{1}-p_{0}\right\|<\left\|q_{1}-p_{0}\right\|_{2}$. By using this result it is straightforward to prove that this heuristic gives always results that are lower then (12).

\section{Fastest approach to $n$ far points with re-entry mission}

As well as the 2 points case, to visit a certain number $n$ of ordered point $q_{1}, q_{2}, \ldots q_{n}$ starting from an initial point $p_{0}$ is not an easy problem.

Let us denote with $d_{i, i+1}$ the Euclidean distance between the point $i$ and the next desired point $i+1$, where for sake of simplicity $d_{0,1}$ denotes the distance between the initial position of the carrier $p_{0}$ and $q_{1}$. An upper bound to the optimal solution can be given for the case when the carried vehicle never takes-off, except to reach the last point, and the carrier itself visits all the points. Such an upper bound has the following total cost

$$
t_{n, u p}=\left[\sum_{i=1}^{n} \frac{d_{i-1, i}}{V_{c}}\right]-\frac{\left(V_{c}+V_{v}\right)}{2 V_{c}} \bar{a}+\frac{\left(V_{c}+V_{v}\right)}{2 V_{v}} \bar{a}
$$

The lower bound can be reached in the same way of the 2 points case by supposing that the carrier-carried couple can be seen as a vehicle able to go at velocity $V_{v}$ for a time $\bar{a}$ for each point to reach. This would mean that the carrier is always able to "cut the edge": just like in the 2 points case this optimal solution is practically reachable only for small angles between the lines connecting 2 consecutive points).
Such a lower bound has the following cost

$$
\begin{aligned}
t_{n, \text { low }} & =\sum_{i=1}^{n-1}\left[\frac{d_{i-1, i}-\bar{a} V_{v}}{V_{c}}+\bar{a}\right]+ \\
& +\left[\frac{d_{n-1, n}}{V_{c}}+\left(\frac{\left(V_{c}+V_{v}\right)}{2} \bar{a}\right)\left(\frac{1}{V_{v}}-\frac{1}{V_{c}}\right)\right] \\
& =\sum_{i=1}^{n-1}\left[\frac{d_{i-1, i}}{V_{c}}\right]+(n-1) \bar{a}\left(1-\frac{V_{v}}{V_{c}}\right)+ \\
& +\left(\frac{\left(V_{c}+V_{v}\right)}{2} \bar{a}\right)\left(\frac{1}{V_{v}}-\frac{1}{V_{c}}\right)
\end{aligned}
$$

While the problem itself can be hard to solve, it is possible to build heuristic solutions able to guarantee some performances. Here we will introduce and discuss one of them based on the 1-point fastest approach with re-entry mission solution. Further possible better heuristic will be only mentioned in this paper and can be build based on the results proposed for the 2 points case.

The basic idea of the "1-step heuristic" is the one of using, at each time step, the 1-step solution: the carrier will proceed straight to the next objective point and the vehicle will take-off only when at a distance $d_{t o}=\left(V_{c}+V_{v}\right) \bar{a} / 2$ from the objective. The carrier will keep going straight while the carried vehicle will visit the point and come back. The rendezvous point will be on the same line at distance $d_{l}=\left(V_{v}-V_{c}\right) \bar{a} / 2$ from the objective. From this rendezvous point the ship will go towards to the next point to visit. By exploiting Carnot theorem, the total cost for such a strategy can be expressed in closed form

$$
\begin{gathered}
t_{n}^{\prime}=\frac{d_{1}-d_{l}+\sum_{i=2}^{n-1}\left[\sqrt{d_{l}^{2}+d_{i}^{2}-2 d_{i} d_{l} \cos \left(\theta_{i}\right)}-d_{l}\right]}{V_{c}}+ \\
+\frac{\sqrt{d_{l}^{2}+d_{n}^{2}-2 d_{n} d_{l} \cos \left(\theta_{i}\right)}-d_{t o}}{V_{c}}+\frac{d_{t o}}{V_{v}}
\end{gathered}
$$

Where $\theta_{i}$ is the angle resulting from the line between $\overline{p_{i-1} p_{i}}$ and $\overline{p_{i} p_{i+1}}$. Simple computations show that the cost (21) has the interesting property of satisfying the following inequality

$$
t_{n, l o w} \leq t_{n}^{\prime} \leq t_{n, u p}
$$

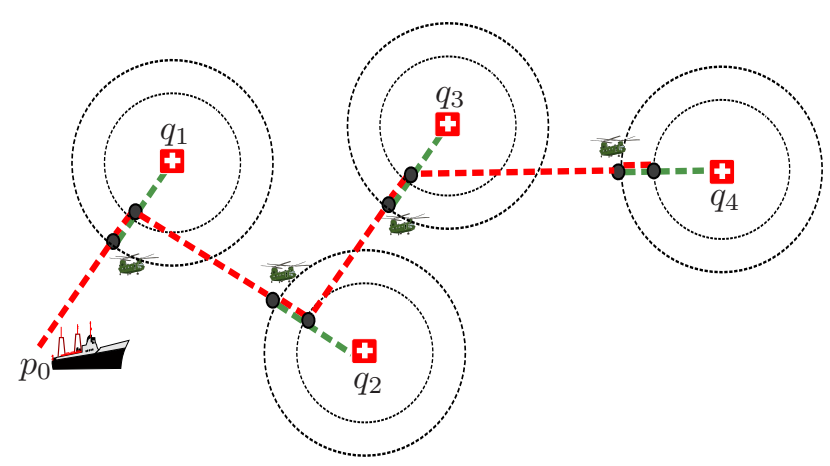

Fig. 8. The geometric interpretation behind the algorithm proposed to visit a set of $n$ (4 in the figure) ordered points.

Inspired by the the results for the 2 points case, we propose also a second euristic solution which is based on the idea of 
satisfying the condition of Proposition 1 by suitably "slow down" the velocity of the vehicle such that the rendezvous is possible. Let us denote with $\theta_{\text {list }}=\left\{\theta_{1}, \theta_{2}, \ldots \theta_{n-1}\right\}$ the set of the $n-1$ angles $\in[0, \pi]$ between the straight lines that connect consecutive points. For each element $i$ of $\theta_{\text {list }}$ we compute the maximum rendezvous velocity $\bar{v}_{i}$ according to the following algorithm

$$
\begin{aligned}
& \text { - } \text { if } \theta_{i} \equiv 0, \bar{v}_{i}=V_{v} \\
& \text { - else } \bar{v}_{i}=\min \left\{V_{v}, \sqrt{\frac{2 V_{c}^{2}}{1-\cos \theta_{i}}}\right\}
\end{aligned}
$$

The maximum rendezvous velocity $\bar{v}_{i}$ is then used in order to bound the velocity of the vehicle to reach the desired point $i$ following the shortest path depicted in Fig. 9. The cost of this solution can be computed as

$$
\begin{aligned}
t_{n}{ }_{n} & =\sum_{i=1}^{n-1}\left[\frac{d_{i-1, i}-\bar{a} \bar{v}_{i}}{V_{c}}+\bar{a}\right]+ \\
& +\left[\frac{d_{n-1, n}}{V_{c}}+\left(\frac{\left(V_{c}+V_{v}\right)}{2} \bar{a}\right)\left(\frac{1}{V_{v}}-\frac{1}{V_{c}}\right)\right]
\end{aligned}
$$

Simple computations shows that the cost (22) verify

$$
t_{n, \text { low }} \leq t_{n}{ }_{n} \leq t_{n, u p}
$$

and moreover if $\bar{v}_{i}=V_{v}$ for all $i=1$,..n-1, which in turns reduces to conditions on the angles and the ratio between the velocity of the carrier and the vehicle, then it coincides with the lower bound (20).

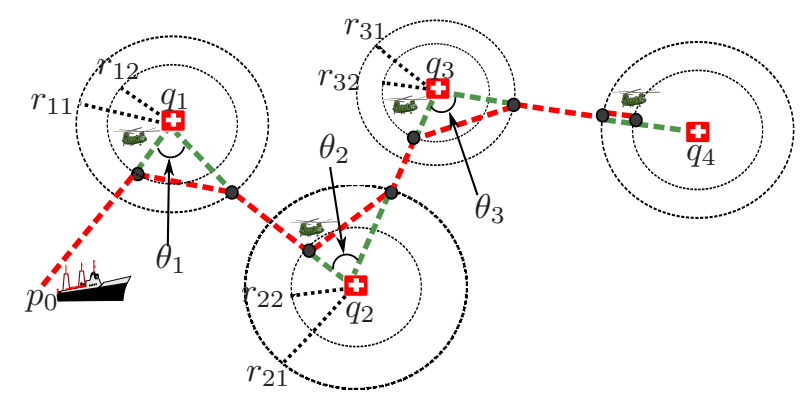

Fig. 9. The geometric interpretation behind the second algorithm proposed to visit a set of $n$ (4 in the figure) ordered points. Notation: $r_{11}=\bar{v}_{1} a_{11}$, $r_{12}=\bar{v}_{1} a_{12}, r_{21}=\bar{v}_{2} a_{21}, r_{22}=\bar{v}_{2} a_{22}, r_{31}=\bar{v}_{3} a_{31}, r_{32}=\bar{v}_{3} a_{31}$, with $a_{i, 1}+a_{i, 2}=\bar{a}$.

\section{Conclusions And Future Work}

In this paper we dealt with the problem of path planning for two complementary vehicles: a slow long-range carrier and a fast vehicle with a limited operativeness. In particular we focused on the "fast rescue problems" i.e. the family of problems in which one has to visit an ordered set of point in minimum time. Several results about optimal solutions and approximation heuristics have been presented. Work is being currently done to extend the results to vehicles with nonholomic constraints.

\section{REFERENCES}

[1] P. R. Chandler, M. Pachter, D. Swaroop, J. M. Fowler, J. K. Howlett, S. Rasmussen, C. Schumacher, and K. Nygard, "Complexity in UAV cooperative control," in American Control Conference, (Anchorage, AK), pp. 1831-1836, 2002.

[2] R. W. Beard, T. W. McLain, M. A. Goodrich, and E. P. Anderson, "Coordinated target assignment and intercept for unmanned air vehicles," IEEE Transactions on Robotics and Automation, vol. 18, no. 6 , pp. 911-922, 2002.

[3] S. Rathinam, R. Sengupta, and S. Darbha, "A resource allocation algorithm for multi-vehicle systems with non holonomic constraints," Tech. Rep. UCB-ITS-RR-2005-2, Institute of Transportation Studies, University of California at Berkeley, May 2005. To appear in IEEE Transactions on Automation Sciences and Engineering.

[4] K. Savla, F. Bullo, and E. Frazzoli, "On traveling salesperson problems for Dubins' vehicle: stochastic and dynamic environments," in IEEE Conf. on Decision and Control, (Seville, Spain), pp. 4530-4535, Dec. 2005.

[5] J. Le Ny and E. Feron, "An approximation algorithm for the curvatureconstrained traveling salesman problem," in 43rd Annual Allerton Conference on Communications, Control and Computing, (Allerton, IL), Sept. 2005 .

[6] S. Darbha, "Combinatorial motion planning for a collection of ReedsShepp vehicles," tech. rep., ASEE/AFOSR SFFP, AFRL, Eglin, Aug. 2005.

[7] E. Frazzoli and F. Bullo, "Decentralized algorithms for vehicle routing in a stochastic time-varying environment," in IEEE Conf. on Decision and Control, (Paradise Island, Bahamas), pp. 3357-3363, Dec. 2004.

[8] A. E. Gil, K. M. Passino, and A. Sparks, "Cooperative scheduling of tasks for networked uninhabted autonomous vehicles," in IEEE Conf. on Decision and Control, (Maui, Hawaii), pp. 522-527, Dec. 2003.

[9] A. Richards, J. Bellingham, M. Tillerson, and J. How, "Coordination and control of multiple UAVs," in AIAA Conf. on Guidance, Navigation and Control, (Monterey, CA), Aug. 2002.

[10] C. Tomlin, I. Mitchell, and R. Ghosh, "Safety verification of conflict resolution manoeuvres," IEEE Transactions on Intelligent Transportation Systems, vol. 2, no. 2, pp. 110-120, 2001.

[11] J.-D. Boissonnat and S. Lazard, "A polynomial-time algorithm for computing a shortest path of bounded curvature amidst moderate obstacles," International Journal of Computational Geometry and Applications, vol. 13, pp. 189-229, 2003.

[12] S. M. LaValle, Planning Algorithms. Cambridge, UK: Cambridge University Press, 2006.

[13] D. J. Bertsimas and G. J. van Ryzin, "A stochastic and dynamic vehicle routing problem in the Euclidean plane," Operations Research, vol. 39 , pp. $601-615,1991$

[14] R.M. Murray, "Recent Research in Cooperative Control of MultiVehicle Systems," in ASME Journal of Dynamic Systems , to appear, 2007

[15] G. Percus and O. C. Martin, "Finite size and dimensional dependence of the Euclidean traveling salesman problem," Physical Review Letters, vol. 76, no. 8, pp. 1188-1191, 1996.

[16] J. J. Enright and E. Frazzoli, "UAV routing in a stochastic time-varying environment," in IFAC World Congress, (Prague, Czech Republic), July 2005. Electronic Proceedings.

[17] S. Lafortune, "Introduction to Discrete Event Systems," in Springer, 2007.

[18] Khler, H. J., Nickel, U., Niere, J., and Zndorf, A., "Integrating UML diagrams for production control systems," in Proceedings of the 22nd international Conference on Software Engineering , (Prague, Czech Republic), July 2000. Electronic Proceedings. 